\title{
Fault Recognition and Diagnosis Based on a Decision Tree for Power Distribution Systems
}

\author{
T C Srinivasa Rao \\ Department of EEE \\ Vasavi College of Engineering \\ Hyderabad, Telangana, India \\ srinivasarao.tc@gmail.com
}

\author{
S S Tulasi Ram \\ Department of EEE \\ J.N.T.U.H. \\ Hyderabad, Telangana, India
}

\author{
J B V Subrahmanyam \\ Department of Electrical Engineering \\ C.J.I.T.S. \\ Jangaon, Telangana, India
}

\begin{abstract}
In recent days, the electrical power system is represented as the complex artificial systems worldwide, as economic and social advancement determined on undamaged, stable, consistent, and economic functions. Due to various arbitrary effects, the unintentional crash happens in electrical power systems. To solve these issues, this paper intends to present the decision tree approach in classifying and detecting fault signals namely sag, transient, and swell in the transmission line. Moreover, the wavelet-decomposed fault signals are extracted and the decision tree is utilized for the diagnoses of fault on the basis of the decomposed signal. Finally, the performance of the proposed approach compares the several existing methods such as SVM, and DBN. The experimental outcomes reveal that the proposed method efficiently notices and classifies the fault signal in the power DS while comparing with the existing methods.
\end{abstract}

Keywords: Power system; Distribution system; fault detection; Wavelet descriptor; Decision tree

\begin{tabular}{ll} 
Nomenclature & \\
\hline Abbreviations & Descriptions \\
\hline DN & Distribution Network \\
DBN & Deep Belief Network \\
SVM & Support Vector Machine \\
DS & Distribution System \\
PCA & Principal Component Analysis \\
FFNN & Feedforward Neural Network \\
WT & Wavelet Transforms \\
MFD & Morphological Fault Detector \\
CNN & Convolutional Neural Network \\
CWT & Continuous Wavelet Transform \\
SPG & Single Phase To Ground \\
RG & Resonant Grounding \\
DSP & Deep Signal Processing \\
FF & Faulty Feeder \\
HIFs & High-Impedance Faults \\
LBFL & Learning-Based Fault Locator \\
ANN & Artificial Neural Network \\
GA & Genetic Algorithm \\
DT & Decision Tree \\
WD & Wavelet Descriptor \\
PCA & Principal Component Analysis \\
\hline
\end{tabular}

\section{Introduction}

The electrical power system comprises a lot of diverse interacting elements and complex dynamic systems that are always prone to an electrical or disturbance fault [9]. The employ of maximum ability electrical generating power plants as well as the idea of grid specifically geographically displaced grids and synchronized electrical power plants. In minimum possible time, it is necessary for the operation of safety equipment and fault detection. As a result, the power system can continue in a stable condition. 
Initially, in the electrical power system transmission lines, the faults occurred are supposed to detect, and subsequently, it needs to classify properly and in the least fast as the possible time, it must be cleared. The security system exploited for a transmission line can also be utilized to start the other relays to secure the power system from outages. A high-quality fault detection system offers an effectual, consistent, rapid as well as a safe method for a relaying operation [10].

In order to guarantee security, dependability and to shun accidents, undesired blackouts and damages to equipment, the fault detection is considered as the significant problem in power systems. There are many kinds of faults that can happen in power systems namely phase to phase, SPG, double phase to ground faults, as well as three phases to ground faults [11][12] [13]. Generally, the SPG fault is considered as one of the most common faults that need to be identified, hence that the faulty section will be isolated quickly.

Basically, in electricity DN, the service discontinuity is considered an issue. Here, fault management and system design are contemplated as core resolution options. As a result, to the growth of fault location approaches, appropriate research efforts have been developed, which are presented to aid usefulness operators in speed up the service restoration as well as minimizing cost and outage time [14]. In [3], classifies the fault placement approaches, which is on the basis of the evaluation of the fault impedance [15], approach that exploit measurements taken along the classical circuit analysis and the system [16], techniques on the basis of traveling waves [17], approaches that exploit the learning theories [18] and methods on the basis of the combination of the preceding schemes.

In many fields, the WT was effectively applied generally; a transformed wave provides an enhanced analysis than the original wave. To extract the fault features the wavelet packet transform is exploited. By the wavelet packet decomposition, it is likely to attain the fault eigenvectors and the ensuing output is exploited as the input of a classifier [19]. However, an additional method, which is conferred, is the ANN that is modeled for drawing a similarity to the operational of the neurons of the brain. The task of a fault classifier is done by ANN and the significance of ANN are better fault classifier cannot be exaggerated. Hence, the ANN is an effectual tool in identifying transmission line faults.

One more current technique of forthcoming the recognition of transmission line fault detection is done by fuzzy logic. The suspicions connected with the recognition of faults are undertaken with the fuzzy logic application to power systems [20] [21] [22]. The employ of fuzzy logic offers a perceptive of the section's likelihood of being a fault in the type of degree of membership and the fault section candidates. Hence, there is a more logical approach than the existing classical set theory. For the transmission line faults identification, the GA is another technique, which can be utilized. GA [24] can give out to be abundant for membership values determination and therefore discovers applicability in power system fault diagnosis.

The main objective of this paper is to present the decision tree method, as the fault diagnosis issue, which is stated as a series of binary classification issue. Moreover, the fault signals in waveletdecomposed is extracted, and the fault signal on the basis of the decomposed signal is also diagnoses in this paper. Hence, the precise fault diagnosis of the power Distributed System is attained by the WD signal and decision tree method.

\section{Literature Review}

In 2018, M. A. Barik et al [1] worked on a decentralized fault detection approach with RG for power distribution systems. Here, the SPG faults were detected and recognized the FF within three cycles of the fault incidence. At first, the faults were noticed on the basis of the displacement of neutral voltage in the presented approach. Subsequently, to recognize the faulty phase the post-fault and pre-fault were exploited. At last, from the link among the faulty phase voltage and the primary transient of the zerosequence current subsequent to the incidence of faults the faulty feeder was recognized. The Mathematical Morphology which was a signal processing tool was used to recognize the FF. To recognize the FF, it was also needed to identify the fault incidence time that was calculated exploiting the slope of the neutral voltage.

In 2018, Mou-Fa Guo, et al [2] proposed a novel technique for FF detection in RG Distributed Systems on the basis of the CWT and CNN. By using the CWT, the time-frequency grayscale images were attained to gather the transient zero-sequence current signals for the sound feeders and faulty feeders. Using CNN, the grayscale image features can be extracted that was trained by a large number of grayscale images in several types of factors and fault conditions.

In 2018, M. Majidi and M. Etezadi-Amoli [3] presented a novel impedance-based approach to place all fault categories with/without distributed generators in DN. Utilizing only the series impedances of the distribution lines was developed, which was the latest method to form an impedance matrix. To calculate the injection fault current, the impedance matrix along with the pre- as well as at-fault voltage phasors 
in some buses were exploited through a least-squares approach. Along with the network if micro-phasor measurement units were installed then linear least-squares estimator was exploited. In the DNs, the operation of the standard protective devices was exploited to decrease the computational burden of the presented technique. In addition, by utilizing the revealed features of the impedance matrix a generalized measurement position method was examined.

In 2018, Moses Kavi et al [4] presented a study on the classification of power system disturbances, which include HIFs. Moreover, the MM approach was utilized by the proposed method. Here, a multistage MFD was formed by utilizing the strategic cascading of suitable filtering functions; also the non-linear MM characteristics were used for the features extraction, which was essential for the characterization of HIFs. The arbitrariness and arc extinction and unsymmetrical/re-ignition characteristics were considered as the target of the HIF features. On the basis of the attributes of power system signals, a weighted convex structuring element was designed.

In 2018, MD Shafiullah and M. A. Abido [5] proposed a new hybrid method, which was the combination of S-Transform and FFNN. In addition, this method was utilized for the classification and detection of distribution grid faults. Here, by S-Transform the measured three-phase current signals were developed with an examination of extracting practical statistical features. To classify and detect various kinds of faults, the extracted features were fetched to FFNN.

In 2018, Miao He and David He [6], presented a novel hybrid DSP technique for bearing fault diagnosis. A deep learning model was formed by entrenched with time-synchronous resampling method, the proposed technique incorporates vibration study methods into deep learning. To examine and reveal the competence of the proposed approach, the data which was gathered from real bearing test rig were exploited.

In 2018, Sekar Kavaskar and Nalin Kant Mohanty [7] proposed a novel detection technique for HIFs on the basis of Mathematical Morphology in power DNs. Here, the current signals were experimental from the distribution feeder to notice HIFs. In a time domain, to extract the features the Mathematical Morphology was exploited, a simple rule-based method was utilized to classify HIFs from other power system disturbances.

In 2019, E. Correa-Tapasco et al [8], presented the adjusting and the validation approach, which was utilized to develop a fault locator. By considering diverse distorted measurements as inputs the performance of the proposed method was examined. The LBFL approach was presented, which was particularly customized for DSs, and its core was the SVM.

\section{Proposed Methodology for Power Sistribution System}

\subsection{System Model}

The system model of classification of transmission signals and fault diagnosis in power system is shown in Fig 1. Here, the features in the signals are extracted using the WD, signal diagnosis, and classification, it is done by utilization of the decision tree. The specimen bus system offers the training signals to be exploited for the proposed method. Actually, the specimen bus system consists of the normal signal and the equivalent fault signals namely sag, transient, as well as swells signals. Each signal WD is evaluated before applying the signals to the proposed method. Furthermore, diagnosis of the fault signal, and the types of signals are classified by the proposed method. 


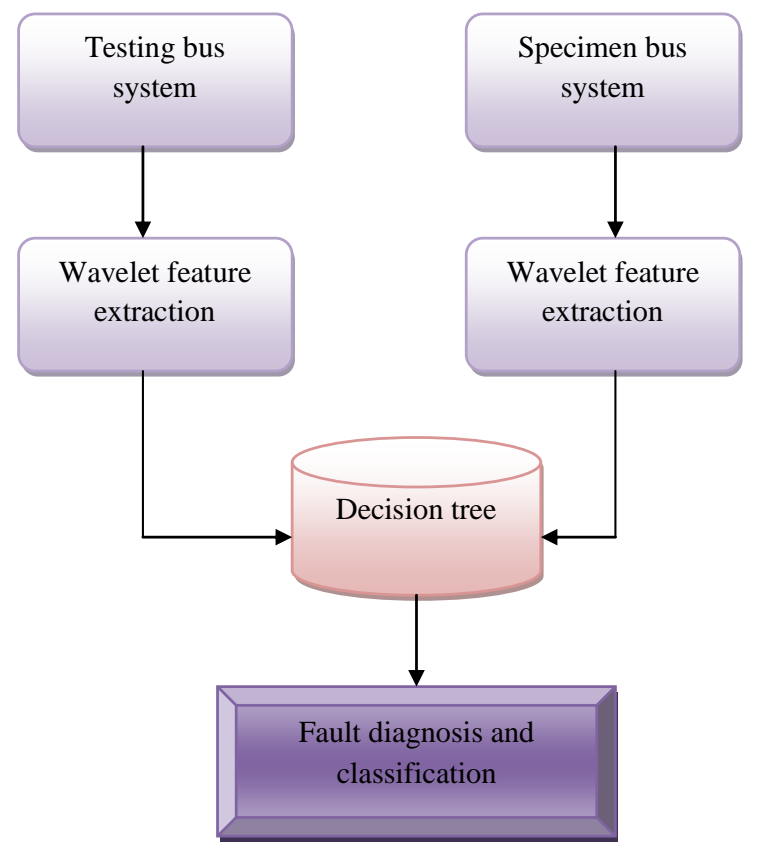

Fig. 1. System model of the proposed classification model and fault diagnosis

\subsection{Signal Decomposition Model}

In a power bus system, the number of transmission lines is diverse with its length. Here, a bus system is considered, which consists of transmission lines with three different distances like $\mathrm{D}_{1}, \mathrm{D}_{2}$ and $\mathrm{D}_{3}$ in $\mathrm{km}$. Each and every transmission line considered its own load, and the position of the fault can also differ. Consequently, each transmission line measurements must be combined with each other before localizing the fault. On the other hand, it is a difficult task to confine the fault happening in such conditions.

Let us consider $\mathrm{x}=\left[\mathrm{u}_{1} ; \mathrm{u}_{2} ; \mathrm{u}_{3} \ldots \ldots \mathrm{u}_{\mathrm{N}}\right]$ as the combined voltage measurements, whereas the signal descriptors are indicated as $\mathrm{u}_{1}, \mathrm{u}_{2}, \mathrm{u}_{3} \ldots \ldots \mathrm{u}_{\mathrm{N}}$ and it represents the number of voltage sources. Let $\mathrm{L}$ be the number of observation instances, $N_{P}$ be the number of observation records, hence the $N_{p} \times L$ represents the size of the combined voltage. Here, the wavelet decomposition of the combined voltage measurements as represented in eq. (1), where the wavelet coefficient stated in eq. (2) stated as $\psi_{\mathrm{p}, \mathrm{q}}(\mathrm{t})$ that exploits two integers namely $\mathrm{p}$ and $\mathrm{q}$ for its explanation, here $\mathrm{p}$ and $\mathrm{q}$ represent the sampling and scaling numbers, correspondingly. The variables $\left(\mathrm{a}_{0}, \mathrm{~b}_{0}\right)$ in eq. $(2)$ describe the constant, which is fixed as $[2,1]$.

$$
\begin{aligned}
& \mathrm{S}(\mathrm{y})=\int_{-\infty}^{\infty} \mathrm{f}(\mathrm{t}) \psi_{\mathrm{p}, \mathrm{q}}^{*}(\mathrm{t}) \mathrm{dt} \\
& \psi_{\mathrm{p}, \mathrm{q}}(\mathrm{t})=\frac{1}{\sqrt{\mathrm{a}_{0}^{\mathrm{p}}}} \psi\left(\frac{\mathrm{t}-\mathrm{qb}_{0} \mathrm{a}_{0}^{\mathrm{p}}}{\mathrm{a}_{0}^{\mathrm{p}}}\right)
\end{aligned}
$$

Furthermore, the size of the decomposed signal indicated as $|\mathrm{S}(\mathrm{y})|^{\mathrm{R}} \times \mathrm{N}_{\mathrm{p}} \times \mathrm{L}$. Here, $|\mathrm{x}|^{\mathrm{R}}$ denotes the number of rows in a matrix x. Since the higher dimension tends to augment the calculation complexity, the $\mathrm{S}(\mathrm{y})$ represents the dimension, which is necessary to minimize. Hence, to minimize the dimension of $\mathrm{S}(\mathrm{y})$, PCA is exploited.

\subsection{Signal descriptors Model}

The signal descriptors model demonstrating fault and normal signals namely swell, transient and sag with its resultant WD portrayed in Fig. 2. Let the time domain signal be $\mathrm{N}$ and the corresponding WD be $\mathrm{N}_{\mathrm{w}}$ that is indicated as $\mathrm{x}$. Moreover, the transient signal is eminent from the normal signal in the area of distorted dimension. The voltage is presumed to be less and then leads to an increase in the particular duration at the first stage. Subsequently, this case remains and leads to minimizing to less value. Additionally, the circumstances augment to raise the voltage subsequent to a definite time that outcomes in creating a normal sine wave. With the reduced voltage, the sag signal starts as well as after that improves with the normal sine wave movement. Furthermore, the feature of the swell signal is relatively greater than the normal voltage, whereas it starts from overvoltage. Since the temporary signal has only 
a small deviation, it is not easy to distinguish the signal's behavior more precisely. Hence, the effectual differentiation of signals can be efficiently performed using the WD.

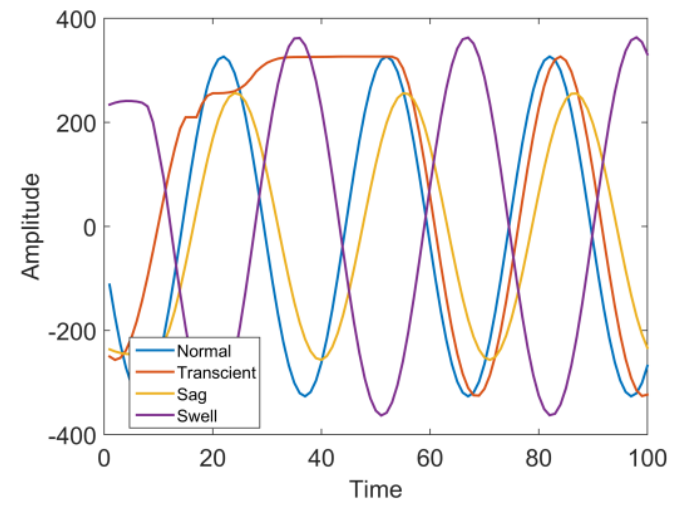

(a)

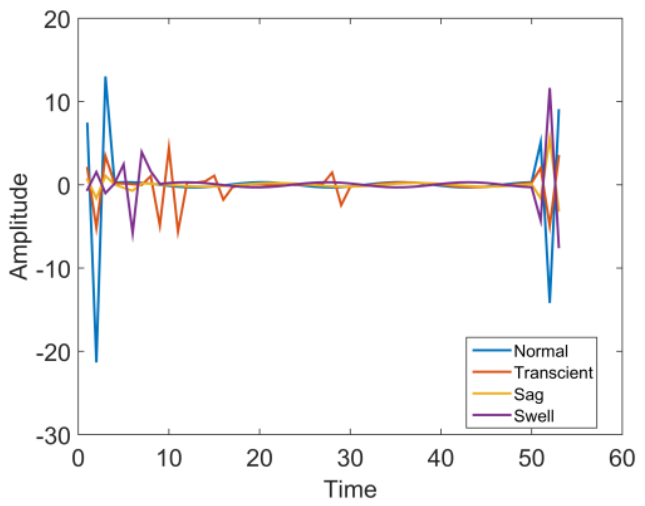

(b)

Fig. 2. Signal descriptor model (a) Temporal signal and (b)Wavelet descriptor

\section{Fault Detection using Decision tree Model}

Generally, DT generation [23] is in a two-phase procedure such as classification and learning. The data, which is collected, are divided into two sub-phases, such as testing phase, and training phase in the learning process. The most significant task of evaluating data mining methods is the creation of a testing phase, and training phase. Generally, in the database, the majority of the data records are randomly chosen for training, as well as the endured data records are exploited for testing. Here, the testing and training phase must arrive from a similar population although must be disjoint. Subsequently, a DT generation approach obtains the training data as input and outputs a DT. Usually exploited DT generation approaches comprise, classification tree and regression trees. In the classification process, at first by making predictions over the test data the precision of attained DT is evaluated. Evaluating the accurate target values of the testing data and the predicted target values, the precision of a decision tree is calculated. If the precision is measured satisfactory, the DT is exploited for the new dataset of the prediction and classification. If not the motivation must be recognized and equivalent solutions must be approved to undertake issues. The steps for generating a DT from the training data are described below:

At first, each and every record in the training data is collected together into a single partition. The approach selects a predictor attribute, which can optimally divide the target class values in the partition at each iteration. The capacity that a predictor attribute can divide the target class values is calculated on the basis of an attribute chosen criteria. Subsequent to a predictor attribute is selected; the approach divides the partition as child partitions to the extent that each child partition comprises the similar value of the chosen attribute. The DT method iteratively divides a partition and halts while any one of the subsequent finishing criteria is met which is stated as below:

1. In a partition, all records contribute to the similar target class value. Hence, the leaf nodes class label is represented by the target class value.

2. Here, the residual predictor attributes are not present, which is exploited to split a more partition. For this condition, the main target class values turn out to be the leaf node label.

3. Moreover, for a particular value of a predictor variable, the records are also not present. For this condition, a leaf node is generated with the main class value in the parent partition.

The DT creation method is a greedy approach. It iteratively divides a partition through selecting a split attribute, which is used to divide the target class values optimally. The option of divide attribute states the DT technique quality and, consequently, the classification precision is based on the potential data. In information theory, the idea of entropy is an extensive reason measure for the DT to characterize the purity of a partition in DT nodes.

Generally, the DT approach is a nonparametric learning method that has the ability to create classifiers regarding a stated issue to infer information for latest unnoticed conditions. A DT has the hierarchical structure of a tree that modelled upside down. The DT model is on the basis of the knowledge base comprising of a huge number of operating points in theory, which covers all the probable conditions of the power system in study. A vector of pre-disturbance steady-state variables termed "attributes", which characterizes each operating points. Moreover, the knowledge base is split in a learning set is exploited in order to derive the classifier structures and a test set utilized to assess the performance of these structures and unobserved operating points. 
At the root node, the decision tree model begins with the complete learning set of pre-classified operating points. These operating points are analyzed to choose the dichotomic test D which divides them best into a number of most "purified," mutually exclusive subsets. For a two-class partition: "secure or insecure" and the test $\mathrm{D}$ is stated as eq. (3)

$$
\mathrm{D}: \mathrm{SA}_{\mathrm{i}}<\mathrm{tv}_{\mathrm{i}}
$$

where $t_{\mathrm{i}}$ represents the best threshold value of the selected attribute $\mathrm{SA}_{\mathrm{i}}$.

The optimal attribute and its threshold value are attained by successively testing the candidate and attribute thresholds and it evaluates their information gain. Hence, the chosen of the best test is on the basis of increasing the extra information gained by the test. On the basis of the entropy of each subset, $\mathrm{E}_{\mathrm{s}}$ represents the information gain in terms of the class partition of its elements is stated in eq. (4).

$$
E_{s}=-\left(f_{r} \log f_{r}+f_{g} \log f_{g}\right)
$$

Where, $f_{g}$ and $f_{r}$ are the relative frequencies of the secure and insecure Operating Points, correspondingly, in the subset. Fig 3 shows the flowchart of the Decision tree.

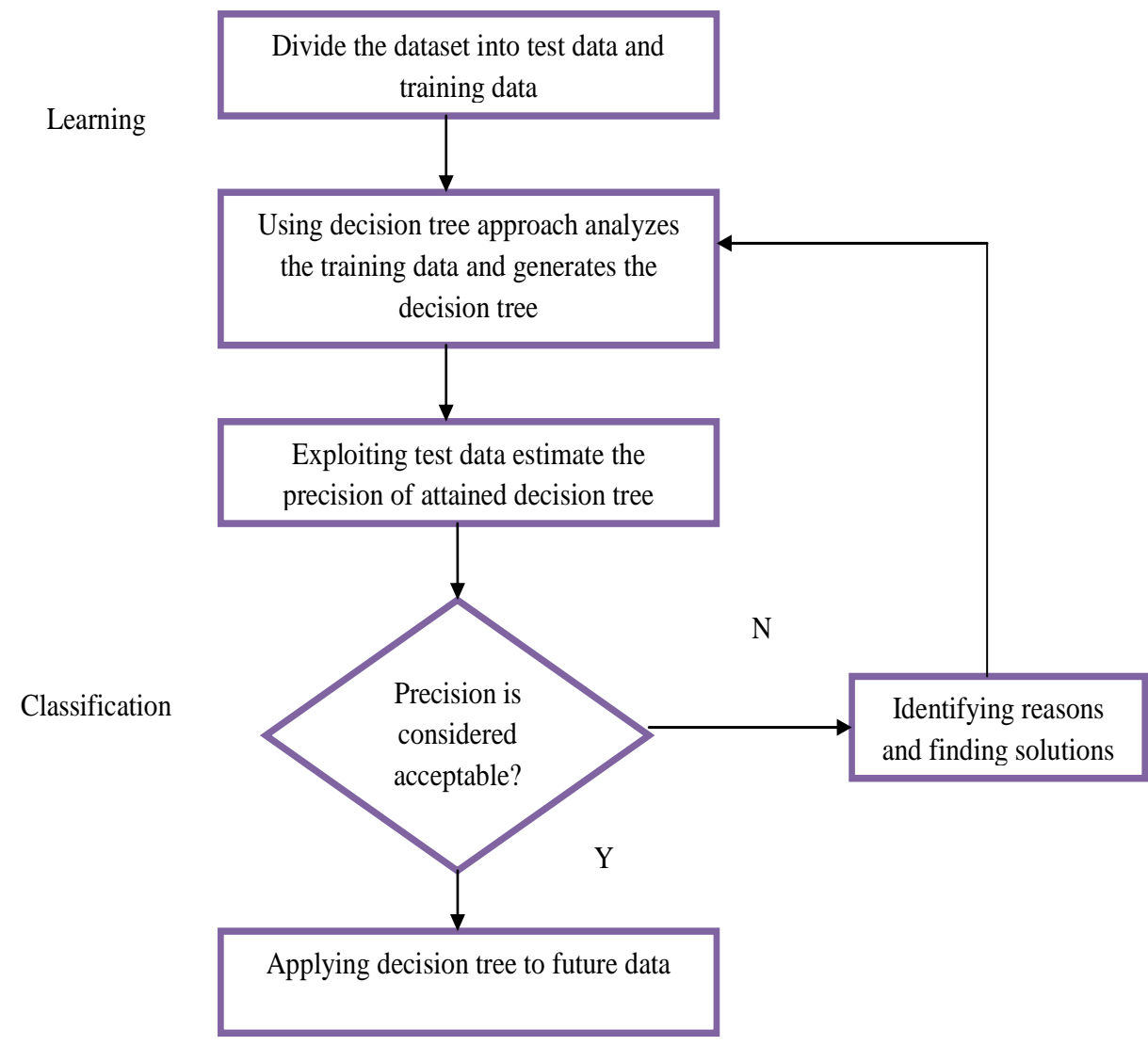

Fig. 3. Flow chart of decision tree

\section{Results and Discussions}

\subsection{Experimental Procedure}

The experimentation in terms of fault signal diagnosis in the DS is experimented in MATLAB and then examines the experimentation outcomes. For the experimentation, $70 \%$ of data is exploited for training and $30 \%$ of data is exploited for testing. Moreover, the performance of the proposed technique is compared to SVM and DBN.

\subsection{Performance Analysis}

In Table 1, 2, 3 and 4 the performance analysis of the proposed method with existing methods such as SVM and DBN is shown for normal signal, transient signal, swell signal and sag signal. In Table 1, the accuracy of the proposed fault recognition model is $38 \%$ better than SVM, $12 \%$ better than the DBN. 
Table 1. Performance analysis of the proposed algorithm with the existing algorithms for normal signal

\begin{tabular}{llll}
\hline Measures & SVM & DBN & Proposed \\
\hline Accuracy & $0.65(3)$ & $0.8(2)$ & $0.9(1)$ \\
Sensitivity & $0(2)$ & $0(2)$ & $0.9(1)$ \\
Specificity & $1(1)$ & $0.9(3)$ & $1(1)$ \\
Precision & $0.25(3)$ & $1(1)$ & $0.25(1)$ \\
FPR & $0(1)$ & $0(1)$ & $0.1(1)$ \\
FNR & $1(3)$ & $0.1(1)$ & $0.1(1)$ \\
NPV & $1(1)$ & $1(1)$ & $1(1)$ \\
FDR & $0(2)$ & $0(2)$ & $0.75(1)$ \\
F1-score & $0(3)$ & $0.95(2)$ & $1.14(1)$ \\
MCC & $0(3)$ & $0.93(2)$ & $1(1)$ \\
G-Mean & $0(3)$ & $0.94(2)$ & $1.15(1)$ \\
Average rank & 2 & 2.2 & 1 \\
Final rank & 3 & 2 & 1 \\
\hline
\end{tabular}

Table 2 summarizes the performance analysis of proposed and existing approaches for recognizing one of the fault signals such as transient signal. Here, the accuracy of the proposed fault recognition model is $32 \%$ superior to SVM, and $12 \%$ superior to DBN method. In the sensitivity measure, the proposed fault recognition model is $67 \%$ superior to SVM, 63\% superior to the DBN model. Similarly, Table 3 depicts the performance analysis on sag signal recognition exploiting both proposed and existing methods. Moreover, the accuracy of the proposed model is $18 \%$ and $23 \%$ outperforms SVM and DBN approaches. In addition, the proposed method obtains high precision, which is superior to the SVM and DBN by $2.22 \%$. Similarly, the NPV of the proposed method is $6.62 \%$ superior to the SVM and $5 \%$ better than DBN.

Table 2. analysis of the proposed method with the existing algorithms for Transient signal

\begin{tabular}{llll}
\hline Measures & SVM & DBN & Proposed \\
\hline Accuracy & $0.95(2)$ & $0.92(3)$ & $1(1)$ \\
Sensitivity & $1(1)$ & $0.9(3)$ & $1(1)$ \\
Specificity & $0.94(3)$ & $0.97(2)$ & $1(1)$ \\
Precision & $0.84(3)$ & $0.9(2)$ & $1(1)$ \\
FPR & $0.07(3)$ & $0.03(2)$ & $0(1)$ \\
FNR & $0(1)$ & $0.1(3)$ & $0(1)$ \\
NPV & $0.94(3)$ & $0.97(2)$ & $1(1)$ \\
FDR & $0.17(3)$ & $0.1(2)$ & $0(1)$ \\
F1-score & $0.91(3)$ & $0.9(2)$ & $1(1)$ \\
MCC & $0.88(3)$ & $0.87(2)$ & $1(1)$ \\
G-Mean & $0.91(2)$ & $0.9(3)$ & $1(1)$ \\
Average rank & 3 & 2 & 1 \\
Final rank & 3 & 2 & 1 \\
\hline
\end{tabular}

Table 3. Performance analysis of the proposed algorithm with the existing algorithms for sag signal

\begin{tabular}{llll}
\hline Measures & SVM & DBN & Proposed \\
\hline Accuracy & $0.95(3)$ & $0.9(2)$ & $0.98(1)$ \\
Sensitivity & $0.9(2)$ & $0.9(2)$ & $1(1)$ \\
Specificity & $0.97(4)$ & $0.9 .3(2)$ & $1(1)$ \\
Precision & $0.9(2)$ & $0.75(3)$ & $1(1)$ \\
FPR & $0.03(3)$ & $0.1(2)$ & $0.3(1)$ \\
FNR & $0.1(2)$ & $0.1(2)$ & $0(1)$ \\
NPV & $0.91(2)$ & $0.9(3)$ & $0(1)$ \\
FDR & $0.1(2)$ & $0.25(3)$ & $0(1)$ \\
F1-score & $0.9(2)$ & $0.82(3)$ & $0.95(1)$ \\
MCC & $0.87(2)$ & $0.76(3)$ & $0.93(1)$ \\
G-Mean & $0.9(1)$ & $0.82(2)$ & $0.9(1)$ \\
Average rank & 3 & 2.4 & 1.4 \\
Final rank & 3 & 2 & 1 \\
\hline
\end{tabular}

Table 4 summarizes the fault signal recognition analysis on swell signal. The proposed method is superior to both the DBN and SVM by $5 \%$ and $7 \%$ in terms of accuracy. Hence, it is stated that the proposed model performs better than when recognizing fault in the power DS. 
Table 4. Performance analysis of the proposed Algorithm with the Existing Algorithms for Swell signal

\begin{tabular}{llll}
\hline Measures & Linear-SVM & RBF-SVM & Quadratic SVM \\
\hline Accuracy & $0.75(3)$ & $1(1)$ & $0.98(2)$ \\
Sensitivity & $0.9(2)$ & $1(1)$ & $0.9(2)$ \\
Specificity & $0.7(2)$ & $1(1)$ & $1(1)$ \\
Precision & $0.5(2)$ & $1(1)$ & $1(1)$ \\
FPR & $0.3(3)$ & $0(1)$ & $0(1)$ \\
FNR & $0.1(2)$ & $0(1)$ & $0.1(2)$ \\
NPV & $0.7(3)$ & $1(1)$ & $1(1)$ \\
FDR & $0.5(2)$ & $0(1)$ & $0(1)$ \\
F1-score & $0.64(2)$ & $1(1)$ & $0.95(3)$ \\
MCC & $0.52(3)$ & $1(1)$ & $0.93(2)$ \\
G-Mean & $0.67(6)$ & $1(1)$ & $0.94 \quad(2)$ \\
Average rank & 3.5 & 1 & 2.2 \\
Final rank & 3 & 1 & 2 \\
\hline
\end{tabular}

\section{Conclusion}

In this paper, the exploit of fault diagnosis techniques in a power DS by the decision tree approach was presented. Moreover, in this experiment, the fault signals have considered such as sag, transient, as well as swell signal. At first, wavelet-decomposed fault signals were extracted, and the next decision tree method was exploited in order to analyze the fault on the basis of the decomposed signal. Moreover, the performance of the decision tree method in identifying fault was compared with conventional methods such as SVM, and DBN approaches. Finally, the decision tree outperforms the conventional methods in diagnosing the fault signals in the power DS.

\section{Compliance with Ethical Standards}

Conflicts of interest: Authors declared that they have no conflict of interest.

Human participants: The conducted research follows the ethical standards and the authors ensured that they have not conducted any studies with human participants or animals.

\section{References}

[1] M. A. Barik, A. Gargoom, M. A. Mahmud, M. E. Haque, H. Al-Khalidi and A. M. Than Oo, "A Decentralized Fault Detection Technique for Detecting Single Phase to Ground Faults in Power Distribution Systems With Resonant Grounding," IEEE Transactions on Power Delivery, vol. 33, no. 5, pp. 2462-2473, Oct. 2018.

[2] M. Guo, X. Zeng, D. Chen and N. Yang, "Deep-Learning-Based Earth Fault Detection Using Continuous Wavelet Transform and Convolutional Neural Network in Resonant Grounding Distribution Systems,"IEEE Sensors Journal, vol. 18, no. 3, pp. 1291-1300, 1 Feb.1, 2018.

[3] M. Majidi and M. Etezadi-Amoli, "A New Fault Location Technique in Smart Distribution Networks Using Synchronized/Nonsynchronized Measurements," in IEEE Transactions on Power Delivery, vol. 33, no. 3, pp. 1358-1368, June 2018.

[4] M. Kavi, Y. Mishra and M. D. Vilathgamuwa, "High-impedance fault detection and classification in power system distribution networks using morphological fault detector algorithm," IET Generation, Transmission \& Distribution, vol. 12, no. 15, pp. 3699-3710, 2882018.

[5] M. Shafiullah and M. A. Abido, "S-Transform Based FFNN Approach for Distribution Grids Fault Detection and Classification," IEEE Access, vol. 6, pp. 8080-8088, 2018.

[6] Miao He, David He,"A new hybrid deep signal processing approach for bearing fault diagnosis using vibration signals",Neurocomputing, In press, corrected proof, 24 April 2019.

[7] Sekar Kavaskar, Nalin Kant Mohanty,"Detection of High Impedance Fault in Distribution Networks",Ain Shams Engineering Journal, vol. 10, no. 1,pp. 5-13, March 2019.

[8] E. Correa-Tapasco, J. Mora-Flórez, S. Perez-Londono,"Performance analysis of a learning structured fault locator for distribution systems in the case of polluted inputs", Electric Power Systems Research, vol. 166, pp. 18, January 2019.

[9] Y. Zhao, Y. An, and Q. Ai, "Research on size and location of distributed generation with vulnerable node identification in the active distribution network," IET Generation, Transmission and Distribution, vol. 8, no. 11, pp. 1801-1809, November 2014.

[10] A. Yadav and A. Swetapadma, "Enhancing the performance of transmission line directional relaying, fault classification and fault location schemes using fuzzy inference system," IET Generation, Transmission and Distribution, vol. 9, no. 6, pp.580-591, April 2015. 
[11] A. Swetapadma and A. Yadav, "Improved fault location algorithm for multi-location faults, transforming faults and shunt faults in thyristor controlled series capacitor compensated transmission line," IET Generation, Transmission and Distribution, vol. 9, no. 13, pp.1597-1607, January 2015.

[12] J.G.M.S. Decanini, M.S.T. Neto, and C.R. Minussi, "Robust fault diagnosis in power distribution systems based on fuzzy ARTMAP neural network-aided evidence theory," IET Generation, Transmission and Distribution, vol. 6, no. 11, pp. 1112-1120, November 2012.

[13] Y. Ma, X. Yu and Y. Niu, "A parallel heuristic reduction based approach for distribution network fault diagnosis," International journal of electrical power and energy systems, vol. 73, pp. 548-559, December 2015.

[14] S. Jamali, A. Bahmanyar, "A new fault location method for distribution networks using sparse measurements," International journal of electrical power and energy systems, vol. 81, pp. 459-468, October 2016.

[15] D.V. Coury, M. Oleskovicz, and R.K. Aggarwal, "An ANN routine for fault detection, classification and location in Transmission lines," Electr. Power Compon. Syst., vol. 30, pp. 1137-1149, 2002.

[16] P. Jena and A.K. Pradhan, "Solution to close-in fault problem in directional relaying," IEEE Trans. Power Deliv., vol. 23, no. 3, pp. 1690-1692, July 2008.

[17] P. Jena and A.K. Pradhan, "An integrated approach for directional relaying of the double-circuit line," IEEE Trans. Power Deliv., vol. 26, no. 3, pp. 1783-1791, July 2011.

[18] M.J. Reddy and D.K.Mohanta, "Adaptive-neuro-fuzzy inference system approach for transmission line fault classification and location incorporating effects of power swing," IET. Gener. Transm. Distrib., vol. 2, no. 2, pp. 235-244, March 2008.

[19] A.J. Mazon, I. Zamora, J.F. Minambres, M. A. Zorrozua, J.J. Barandiaran, and K. Sagastabeitia, "A new approach to fault location in two-terminal transmission lines using artificial neural networks," Electr. Power Syst. Res. J., vol. 56, no. 3, pp. 261-266, December 2000.

[20] Z.Q. Bo, R.K. Aggarwal, A.T. Johns, H.Y. Li, and Y.H. Song, "A new approach to phase selection using fault generated high frequency noise and neural networks," IEEE Trans. Power Deliv., vol. 12, no. 1, pp. 106-115, January 1997.

[21] B. Das and J.V. Reddy, "Fuzzy-logic-based fault classification scheme for digital distance protection," IEEE Trans. Power Deliv.,vol. 20, no. 2, pp. 609-616, April 2005.

[22] S.R. Samantaray, "A Systematic fuzzy rule based approach for fault classification in transmission lines," Appl. Soft Comput., vol. 13, pp. 928-938, February 2013.

[23] Yuanhong Li, Ming Dong and R. Kothari, "Classifiability-based omnivariate decision trees," IEEE Transactions on Neural Networks, vol. 16, no. 6, pp. 1547-1560, Nov. 2005.

[24] RT Satish, KT Reddy, "A technique to find optimal location for wavelet-based image watermarking using genetic algorithm", Machine Graphics \& Vision International Journal,vol. 20 (2), pp.173-196, 2011. 